\title{
Quasi-Linear Convection-Dominated Transport Problem Based on Characteristics-Mixed Finite Element Method
}

\author{
Peixin Zhao ${ }^{1}$ and Hongying Man $^{2}$ \\ ${ }^{1}$ School of Management, Shandong University, Jinan, Shandong 250100, China \\ ${ }^{2}$ Department of Mathematics, Beijing Institute of Technology, Beijing 100081, China \\ Correspondence should be addressed to Peixin Zhao; pxzhao@126.com
}

Received 3 July 2013; Accepted 4 August 2013

Academic Editor: Shurong Sun

Copyright (C) 2013 P. Zhao and H. Man. This is an open access article distributed under the Creative Commons Attribution License, which permits unrestricted use, distribution, and reproduction in any medium, provided the original work is properly cited.

\begin{abstract}
We propose the characteristics-mixed method for approximating the solution to a convection-dominated transport problem. The new method is a combination of characteristic approximation to handle the convection part in time and a mixed finite element spatial approximation to deal with the diffusion part. Boundary conditions are incorporated in a natural fashion. The scheme is locally conservative since fluid is transported along the approximate characteristics on the discrete level and the test function can be piecewise constant. Analysis shows that the scheme has much smaller time-truncation errors than those of standard methods.
\end{abstract}

\section{Introduction}

Given an open bounded domain $\Omega \subset R^{2}$ with a smooth boundary $\Gamma$ and a time interval $(0, T]$, we consider in this paper the following convection-diffusion equation:

(a) $\frac{\partial c}{\partial t}+u \cdot \nabla c-\nabla \cdot(a(c) \nabla c)=f(c)$ in $\Omega \times(0, T]$

(b) $\frac{\partial c}{\partial n}=0 \quad$ on $\Gamma \times(0, T]$,

(c) $c(x, 0)=c_{0}(x)$ in $\Omega$,

where

(1) $c(x, t)$ denotes, for example, the concentration of a possible substance;

(2) $u(x)$ represents the velocity of the flow;

(3) $\nabla$ and $\nabla \cdot$ denote the gradient and the divergence operators, respectively;

(4) $a(c)$ is sufficiently smooth and there exist constants $a_{1}$ and $a_{2}$ such that $0<a_{1} \leq a(c) \leq a_{2}<+\infty$;

(5) $f$ denotes a source term.

This equation governs such phenomena as the flow of heat within a moving fluid, the transport of dissolved nutrients or contaminants within the groundwater, and the transport of a surfactant or tracer within an incompressible oil in a petroleum reservoir.

For convenience, we assume (1) is $\Omega$-periodic, this is physically reasonable since no-flow boundaries are generally treated by reflection, and because in general interior flow patterns are much more important than boundary effects. Thus, the no-flow boundary condition above can be dropped. Because of molecular diffusion, $a(c)$ is uniformly positive. Although this implies that the equation is uniformly parabolic, in many applications the Peclet number is quite high. Thus, convection dominates diffusion and the equation is nearly hyperbolic in nature. The concentration often develops sharp fronts that are nearly shocking. It is well known that strictly parabolic discretization schemes applied to the problem do not work well when it is convection dominated. It is especially difficult to approximate well the sharp fronts and conserve the material or mass in the system.

Effective discretization schemes should recognize to some extent the hyperbolic nature of the equation. Many such schemes have been developed, such as the explicit method of characteristics, upstream-weighted finite difference schemes [1], higher-order Godunov schemes [2, 3], the streamline diffusion method [4], the least-squares-mixed finite element method $[5,6]$, the modified method of 
characteristics-Galerkin finite element procedure (MMOCGalerkin) [7-9], and the Eulerian-Lagrangian localized adjoint method (ELLAM) [10]. Each method has its advantages and disadvantages. Explicit characteristic and Godunov schemes require that a Courant-Friedrich-Lewy (CFL) time step constraint is to be imposed. Upstream weighting tends to introduce into the solution an excessive amount of numerical diffusion near the sharp fronts. Compared with upstream weighting, the streamline diffusion method and the leastsquares-mixed finite element method reduce the amount of numerical diffusion. It adds a user-defined amount of bias in the direction of streamline. In ELLAM, it can be difficult to evaluate the resulting integrals.

We concentrate on MMOC-Galerkin. It is an implicit scheme, so reasonably large time steps may be used, and it does not numerically diffuse the fronts to a particularly excessive degree. Unfortunately, it has certain inherent difficulties, especially with regard to local mass balance.

Mixed finite element method has been found to be very useful for solving flow equations not only for its optimal approximation of the unknown function and the vector flux, but also for its mass conservation due to the use of piecewise test function. Its theories and applications have been extensively discussed, for example, see [11-14].

Therefore, to purse the high performance of an algorithm, one should take a nice combination of MMOC and the mixed finite element method. In this way, characteristicsmixed finite element method was considered. However, most integrals in this method involve the transformation images of the test function, so it is difficult to compute in practice.

In this paper, we propose a new characteristics-mixed finite element scheme. It is similar to MMOC-Galerkin in that we approximate the hyperbolic part of the equation along the characteristics. We use, however, a mixed finite element spatial discretization of the equation. Since piecewise constant functions can be used as test functions, the scheme conserves mass locally and globally in the discrete level. One of our goals in the paper is to yield $K\left\|\partial^{2} c / \partial \tau^{2}\right\| \Delta t$ in time truncation. Since the solution changes much less rapidly in the characteristic direction, the bound in time truncation will permit the use of larger time steps, with corresponding improvements in efficiency, at no cost in accuracy. For we use the mixed finite element, we can simultaneously approximate the scalar unknown and the vector flux. In error analysis, we introduce some new techniques to overcome difficulties in dealing with the combination of MMOC and the mixed finite element. The optimal error estimates for the unknown function and the vector flux are given.

An outline of the paper is as follows. In Section 2, we define an approximation to the characteristics and the characteristics-mixed finite element formulation of the problem. We give the proof of the existence and uniqueness of the discrete problem in Section 3. Several important lemmas are given in Section 4, the critical argument in this section is the proof that for any $\eta \in L^{2}(\Omega),\left|\left(\eta-\bar{\eta}, e_{h}\right)\right|$ is bounded by a constant multiple of $(h+\Delta t)\|\eta\|\left(\left\|\varepsilon_{h}\right\|+\left\|e_{h}\right\|+\|\rho\|\right)$. The optimal order estimates for $c-c_{h}, p-p_{h}$ in $L^{2}(\Omega)$ are presented in Section 5.

\section{The Characteristics-Mixed Finite Element Formulation}

We begin this section by introducing some notations. We denote by $W^{k, p}(S)$ the standard Sobolev space of $k$ differential functions in $L^{p}(S)$. Let $\|\cdot\|_{k, p, S}$ be its norm and let $\|\cdot\|_{k, S}$ be the norm of $H^{k}(S)=W^{k, 2}(S)$ or $H^{k}(S)^{2}$, where we omit $S$ if $S=\Omega$. When $k=0$, we let $L^{2}$ denote the corresponding space defined on $\Omega$ and $\|\cdot\|$ let denote its norm.

We also use the following spaces that incorporate time dependence. If $[a, b] \subset[0, T], X$ is a Sobolev space and $f(x, t)$ suitably smooths on $\Omega \times[a, b]$, we let

$$
\begin{gathered}
L^{p}(a, b ; X)=\left\{f: \int_{a}^{b}\|f(\cdot, t)\|_{X}^{p} d t<\infty\right\}, \\
\|f\|_{L^{p}(a, b ; X)}=\left(\int_{a}^{b}\|f(\cdot, t)\|_{X}^{p} d t\right)^{1 / p},
\end{gathered}
$$

where if $p=\infty$, the integral is replaced by the essential supremum.

We list the assumptions about the coefficients as follows:

$$
\begin{gathered}
u \in W^{1, \infty}(\Omega), \\
\left|\frac{\partial a(c)}{\partial c}\right|+\left|\frac{\partial^{2} a(c)}{\partial c^{2}}\right|+\left|\frac{\partial f(c)}{\partial c}\right| \leq K
\end{gathered}
$$

here and throughout this paper, $K$ denotes different constants in different places.

We also assume that the solution $c$ of (1) satisfies the following:

$$
\begin{aligned}
& \text { (a) } c \in L^{\infty}\left(0, T ; H^{k+1}(\Omega)\right), \\
& \text { (b) } c_{t} \in L^{2}\left(0, T ; H^{k+1}(\Omega)\right) \bigcap L^{\infty}\left(0, T ; L^{\infty}\right), \\
& \text { (c) } c_{t t} \in L^{2}\left(0, T ; L^{2}(\Omega)\right) .
\end{aligned}
$$

Under the above assumptions, we begin to discretize the problem. Let $\psi(x)=\left(1+|u|^{2}\right)^{1 / 2}$ and let the characteristic direction associated with the operator $c_{t}+u \cdot \nabla c$ be denoted by $\tau=\tau(x)$, where

$$
\frac{\partial}{\partial \tau(x)}=\frac{1}{\psi(x)} \frac{\partial}{\partial t}+\frac{u}{\psi(x)} \cdot \nabla,
$$

then, (1)(a) can be put in the form the following:

$$
\psi(x) \frac{\partial c}{\partial \tau}-\nabla \cdot(a(c) \nabla c)=f(c), \quad(x, t) \in \Omega \times(0, T] .
$$

Define the spaces: $V=L_{2}(\Omega), H=\{\chi \in H(\operatorname{div} ; \Omega) ; \chi$. $\vec{n}=0$ on $\Gamma\}$ and let $p=a(c) \nabla c$, then the mixed variational problem corresponding to (1) is to find $(c, p):[0, T] \rightarrow V \times$ $H$, such that

$$
\begin{aligned}
& \text { (a) }\left(\psi(x) \frac{\partial c}{\partial \tau}, v\right)-(\operatorname{div} p, v)=(f, v) \quad \forall v \in V, \\
& \text { (b) }(\alpha(c) p, \chi)+(c, \operatorname{div} \chi)=0 \quad \forall \chi \in H, \\
& \text { (c) } c(x, 0)=c_{0}(x) \quad \forall x \in \Omega,
\end{aligned}
$$

where $\alpha(c)=1 / a(c) \geq a_{0}>0$ and it is this form that will be discretized below. 
Remark 1. The restriction $\chi \cdot \vec{n}=0$ on the space $H$ can be removed if the Dirichlet boundary condition is imposed, that is, the results presented in the following section also hold in the case of the first or the second type boundary value problems.

Let $T_{h}$ be a quasi-regular polygonaization of $\Omega$ and let $V_{h} \times H_{h} \subset V \times H$ be the associated Raviart-Thomas-Nedelec space [15] of index $k \geq 0$. In the procedure to be used, we will consider a time step $\Delta t>0$ and approximate the solution at times $t^{n}=n \Delta t$. The characteristic derivative will be approximated basically in the following manner.

Let $\bar{x}=x-u(x) \Delta t$ and note that

$$
\begin{aligned}
\left.\psi(x) \frac{\partial c}{\partial \tau}\right|_{t_{n}} & \approx \psi(x) \frac{c\left(x, t^{n}\right)-c\left(\bar{x}, t^{n-1}\right)}{\sqrt{(x-\bar{x})^{2}+(\Delta t)^{2}}} \\
& =\frac{c\left(x, t^{n}\right)-c\left(\bar{x}, t^{n-1}\right)}{\Delta t},
\end{aligned}
$$

so, our characteristics-mixed finite element method is the determination of the map $\left(c_{h}, p_{h}\right):\left\{t^{0}, t^{1}, \ldots, t^{N}\right\} \rightarrow V_{h} \times$ $H_{h}$ satisfying the relations following:

(a) $\left(\frac{c_{h}^{n}-\bar{c}_{h}^{n-1}}{\Delta t}, v_{h}\right)-\left(\operatorname{div} p_{h}^{n}, v_{h}\right)$

$$
=\left(f\left(c_{h}^{n}\right), v_{h}\right) \quad \forall v_{h} \in V_{h}, 1 \leq n \leq N,
$$

(b) $\left(\alpha\left(c_{h}^{n}\right) p_{h}^{n}, \chi_{h}\right)+\left(c_{h}^{n}, \operatorname{div} \chi_{h}\right)=0 \quad \forall \chi_{h} \in H_{h}, 0 \leq n \leq N$,

(c) $c_{h}^{0}=\widetilde{c}_{0}$,

where $c_{h}^{n}=c_{h}\left(t^{n}\right)$, and $\bar{c}_{h}^{n-1}=c_{h}^{n-1}(\bar{x})=c_{h}^{n-1}(x-u(x) \Delta t), \widetilde{c}_{0}$ are defined in (18) for $t=0$.

Remark 2. This scheme is an implicit scheme and it is difficult to compute in practice. For simplicity, we can use $f\left(c_{h}^{n-1}\right), \alpha\left(c_{h}^{n-1}\right)$ instead of $f\left(c_{h}^{n}\right), \alpha\left(c_{h}^{n}\right)$. Then and (10) becomes an explicit scheme, but the following results still hold.

\section{The Existence and Uniqueness of the Solution of the Discrete Problem}

In this section, we give the proof of the existence and uniqueness of the solution of the discrete problem (10).

Theorem 3. There exists a unique solution $\left(c_{h}^{n}, p_{h}^{n}\right) \in V_{h} \times H_{h}$ to the characteristics-mixed finite element procedure (10).

Proof. Let $\left\{\phi_{i}\right\}_{i=1}^{M} \subset V_{h}$ and $\left\{\psi_{i}\right\}_{i=1}^{N} \subset H_{h}$ be two sets of bases, respectively, and let

$$
c_{h}^{n}=\sum_{i=1}^{M} C_{i}^{n} \phi_{i}, \quad p_{h}^{n}=\sum_{j=1}^{N} P_{j}^{n} \psi_{j},
$$

then (10) may be written in the following matrix form.
Find

$$
C^{n}=\left(C_{1}^{n}, C_{2}^{n}, \ldots, C_{M}^{n}\right)^{T}, \quad P^{n}=\left(P_{1}^{n}, P_{2}^{n}, \ldots, P_{N}^{n}\right)^{T},
$$

such that

$$
\begin{aligned}
& \text { (a) } A C^{n}-\Delta t B P^{n}=\Delta t F\left(C^{n}\right)+G^{n-1} \text {, } \\
& \text { (b) } D\left(C^{n}\right) P^{n}+B^{T} C^{n}=0,
\end{aligned}
$$

where

$$
\begin{gathered}
A=\left(\left(\phi_{i}, \phi_{j}\right)\right)_{M \times M}, \\
D\left(C^{n}\right)=\left(\left(\alpha\left(c_{h}^{n}\right) \psi_{i}, \psi_{j}\right)\right)_{N \times N^{\prime}} \\
B=\left(\left(\phi_{i}, \operatorname{div} \psi_{j}\right)\right)_{M \times N^{\prime}} \\
F\left(C^{n}\right)=\left(\begin{array}{c}
\left(f\left(c_{h}^{n}\right), \phi_{1}\right) \\
\vdots \\
\left(f\left(c_{h}^{n}\right), \phi_{M}\right)
\end{array}\right), \\
G^{n-1}=\left(\begin{array}{c}
\left(\bar{c}_{h}^{n-1}, \phi_{1}\right) \\
\vdots \\
\left(\bar{c}_{h}^{n-1}, \phi_{M}\right)
\end{array}\right) .
\end{gathered}
$$

Since $\left\{\phi_{i}\right\}_{i=1}^{M}$ and $\left\{\psi_{i}\right\}_{i=1}^{N}$ are bases, respectively, and $\alpha\left(c_{h}^{n}\right) \geq a_{0}$, $A$ and $D$ are two symmetric positive definite matrices. Solve $P^{n}$ from (13)(b), then substitute it into (13)(a), we have

$$
A C^{n}+\Delta t B\left(D\left(C^{n}\right)\right)^{-1} B^{T} C^{n}=\Delta t F\left(C^{n}\right)+G^{n-1}
$$

Then,

$$
A C^{n}=G^{n-1}+\widetilde{F}\left(C^{n}\right) \Delta t
$$

where

$$
\widetilde{F}\left(C^{n}\right)=F\left(C^{n}\right)-B\left(D\left(C^{n}\right)\right)^{-1} B^{T} C^{n} .
$$

Since $a(c)$ and $f(c)$ are globally Lipschitz continuous about $c, \widetilde{F}$ is globally Lipschitz continuous about $C^{n}$ too. With $C^{0}$ given by (10)(c) it is clear that this nonlinear system of algebraic equations may be solved for small $\Delta t$, so that (10) defines a unique discrete solution for $n \Delta t \leq T$.

In order to analysis the convergence of the method, it is convenient to introduce the mixed elliptic projection associated with our equations.

Let $(\widetilde{c}, \widetilde{p}):[0, T] \rightarrow V_{h} \times H_{h}$ be given by the following relations

$$
\begin{aligned}
& \text { (a) }-\left(\operatorname{div}(p-\tilde{p}), v_{h}\right)=0, \\
& \forall v_{h} \in V_{h}, 0 \leq t \leq T, \\
& \text { (b) }\left(\alpha(c)(p-\widetilde{p}), \chi_{h}\right)+\left(c-\widetilde{c}, \operatorname{div} \chi_{h}\right)=0, \\
& \forall \chi_{h} \in H_{h}, 0 \leq t \leq T .
\end{aligned}
$$


Let $e_{h}^{n}=c_{h}^{n}-\widetilde{c}^{n}, \rho^{n}=\widetilde{c}^{n}-c^{n}, \varepsilon_{h}^{n}=p_{h}^{n}-\widetilde{p}^{n}$, and $\sigma^{n}=$ $\tilde{p}^{n}-p^{n}$. By [11] we know that for $p=2$ or $+\infty$ and $1 \leq s \leq k+1$, consider

$$
\begin{gathered}
\|\rho\|_{L^{p}\left(0, T ; L^{2}\right)} \leq K\|c\|_{L^{p}\left(0, T ; H^{\left.s+\delta_{0 k}\right)}\right.} h^{s}, \\
\|\sigma\|_{L^{p}\left(0, T ; L^{2}\right)} \leq K\|c\|_{L^{p}\left(0, T ; H^{s+1}\right)} h^{s} .
\end{gathered}
$$

It follows easily that

$\left\|\rho_{t}\right\|_{L^{p}\left(0, T ; L^{2}\right)}$

$$
\leq K\left(\|\mathcal{c}\|_{L^{p}\left(0, T ; H^{\left.s+\delta_{0 k}\right)}\right.}+\left\|\mathcal{c}_{t}\right\|_{L^{p}\left(0, T ; H^{\left.s+\delta_{0 k}\right)}\right.}\right) h^{s} .
$$

We also have

$$
\|\widetilde{p}\|_{L^{\infty}\left(0, T ; L^{\infty}\right)} \leq K, \quad\left\|\widetilde{p}_{t}\right\|_{L^{\infty}\left(0, T ; L^{\infty}\right)} \leq K .
$$

By (8), (10), and (18), we obtain the error equation in the following form

$$
\begin{aligned}
& \text { (a) }(\left.\frac{e_{h}^{n}-\bar{e}_{h}^{n-1}}{\Delta t}, v_{h}\right)-\left(\operatorname{div} \varepsilon_{h}^{n}, v_{h}\right) \\
&=\left(\psi \frac{\partial c^{n}}{\partial \tau}-\frac{c^{n}-\bar{c}^{n-1}}{\Delta t}, v_{h}\right)-\left(\frac{\rho^{n}-\bar{\rho}^{n-1}}{\Delta t}, v_{h}\right) \\
&+\left(f\left(c_{h}^{n}\right)-f\left(c^{n}\right), v_{h}\right), \quad \forall v_{h} \in V_{h}, \\
& \text { (b) }\left(\alpha\left(c_{h}^{n}\right) \varepsilon_{h}^{n}, \chi_{h}\right)+\left(e_{h}^{n}, \operatorname{div} \chi_{h}\right) \\
&=\left(\left(\alpha\left(c^{n}\right)-\alpha\left(c_{h}^{n}\right)\right) \widetilde{p}^{n}, \chi_{h}\right), \quad \forall \chi_{h} \in H_{h} .
\end{aligned}
$$

\section{Several Lemmas}

To obtain the optimal error estimates, we introduce the following three basic lemmas. These three lemmas are crucial to our main arguments.

Lemma 4. For any function $\tau \in L^{2}(\Omega)$, there exists a function $q_{\tau} \in H^{1}(\Omega)^{2}$, such that
(a) $\operatorname{div} q_{\tau}=\tau$,
(b) $\left\|q_{\tau}\right\|_{1} \leq K\|\tau\|$,
(c) $\left\|q_{\tau}\right\| \leq K\|\tau\|_{-1}$,

where $K$ is a constant.

Lemma 5. For any $\tau \in L^{2}(\Omega)$, if $\left(e_{h}, \varepsilon_{h}\right) \in V_{h} \times H_{h}$ is satisfying

$$
\begin{aligned}
& \left(\alpha \varepsilon_{h}, q_{h}\right)+\left(e_{h}, \operatorname{div} q_{h}\right) \\
& \quad=\left(\left(\alpha\left(c_{1}\right)-\alpha\left(c_{2}\right)\right) \widetilde{p}, q_{h}\right), \quad \forall q_{h} \in H_{h},
\end{aligned}
$$

then, there exists a constant $K>0$ such that

$$
\left|\left(\tau, e_{h}\right)\right| \leq K\left(h\|\tau\|+\|\tau\|_{-1}\right)\left(\left\|\varepsilon_{h}\right\|+\left\|c_{1}-c_{2}\right\|\right) .
$$

Lemma 6. Let $\eta \in L^{2}(\Omega), \bar{\eta}=\eta(x-u(x) \Delta t), u \in W^{1, \infty}(\Omega)$. If $\left(e_{h}, \varepsilon_{h}\right) \in V_{h} \times H_{h}$ satisfying

$$
\begin{aligned}
& \left(\alpha \varepsilon_{h}, q_{h}\right)+\left(e_{h}, \operatorname{div} q_{h}\right) \\
& \quad=\left(\left(\alpha\left(c_{1}\right)-\alpha\left(c_{2}\right)\right) \tilde{p}, q_{h}\right), \quad \forall q_{h} \in H_{h},
\end{aligned}
$$

Then,

$$
\left|\left(\eta-\bar{\eta}, e_{h}\right)\right| \leq K(h+\Delta t)\|\eta\|\left(\left\|\varepsilon_{h}\right\|+\left\|c_{1}-c_{2}\right\|\right) .
$$

Proof. Let $\tau=\eta-\bar{\eta} \in L^{2}(\Omega)$. By Lemma 5 we have

$$
\begin{aligned}
& |(\eta-\bar{\eta}, \xi)| \\
& \quad \leq K\left(h\|\eta-\bar{\eta}\|+\|\eta-\bar{\eta}\|_{-1}\right)\left(\left\|\varepsilon_{h}\right\|+\left\|c_{1}-c_{2}\right\|\right) .
\end{aligned}
$$

To obtain the desired estimate, what we should deduce is to demonstrate the following:

$$
\begin{gathered}
\|\eta-\bar{\eta}\|_{-1} \leq K\|\eta\| \Delta t, \\
\|\eta-\bar{\eta}\| \leq K\|\eta\| .
\end{gathered}
$$

Thus, consider

$$
\begin{gathered}
\|\eta-\bar{\eta}\|_{-1}=\sup _{g \in H^{1}(\Omega)}\left[\frac{1}{\|g\|_{1}} \int_{\Omega}(\eta(x)-\eta(x-u(x) \Delta t))\right. \\
\times g(x) d x] .
\end{gathered}
$$

About the transformation $z=H(x)=x-u(x) \Delta t$, because of the smoothness and periodicity of $u, H$ is a differentiable homeomorphism of $\Omega$ onto itself for $\Delta t$ sufficiently small (see [9]). By using the Jacobian of this transformation and a change of variables, we have

$$
\begin{aligned}
\|\eta-\bar{\eta}\|_{-1} \leq \sup _{g \in H^{1}(\Omega)}\left[\frac{1}{\|g\|_{1}} \int_{\Omega} \eta(x) g(x)\right. \\
\left.\times\left(1-\operatorname{det}\left(D H^{-1}(x)\right)\right) d x\right] \\
+\sup _{g \in H^{1}(\Omega)}\left[\frac{1}{\|g\|_{1}} \int_{\Omega} \eta(z)\right. \\
\times\left(g(z)-g\left(H^{-1}(z)\right)\right) \operatorname{det} \\
\left.\quad \times\left(D H^{-1}(z)\right) d z\right] \\
=W_{1}+W_{2} .
\end{aligned}
$$

It is easy to obtain

$$
\left|W_{1}\right| \leq K \sup _{g \in H^{1}(\Omega)}\left[\frac{\|\eta\|\|g\|}{\|g\|_{1}} \Delta t\right] \leq K\|\eta\| \Delta t .
$$


Next, let $\vec{l}$ be the unit vector in the direction of $z-H^{-1}(z)$; then

$$
\begin{aligned}
\left|W_{2}\right| \leq K \sup _{g \in H^{1}(\Omega)}\left[\frac{1}{\|g\|_{1}}\right. & \quad \times \int_{\Omega} \eta(z) \int_{0}^{1} \frac{\partial g}{\partial \vec{l}}\left((1-\theta) H^{-1}(z)+\theta z\right) d \theta \\
& \left.\times\left|z-H^{-1}(z)\right| d z\right] \\
\leq K \sup _{g \in H^{1}(\Omega)}\left[\frac{\int_{\Omega} \eta(z) d z\|g\|_{1} \Delta t}{\|g\|_{1}}\right] &
\end{aligned}
$$

We now obtain (30) by combining (33), (34), and (36). A similar change of variables demonstrates that

$$
\|\bar{\eta}\|^{2} \leq(1+K \Delta t)\|\eta\|^{2}
$$

Then it is easy to obtain

$$
|(\eta-\bar{\eta}, \xi)| \leq K(h+\Delta t)\|\eta\|\left(\left\|\varepsilon_{h}\right\|+\left\|c_{1}-c_{2}\right\|\right) .
$$

\section{Error Estimates}

Under the above assumptions about $\Omega, u, c$, and $f$, we can derive the optimal order estimates of $\left(c_{h}-c\right)$ and $\left(p_{h}-p\right)$.

Theorem 7. Let $\left(c_{h}, p_{h}\right),(c, p)$ denote the solution of (10) and (8), respectively. Suppose that $\Delta t=O(h)$, we have for sufficiently small $\Delta t>0$ the following:

$$
\begin{aligned}
\max _{0 \leq n \leq N} & \left\|\left(c_{h}-c\right)\left(t^{n}\right)\right\| \\
\leq & K\left(\|c\|_{L^{\infty}\left(0, T ; H^{\left.k+1+\delta_{0 k}\right)}\right.}+\left\|c_{t}\right\|_{L^{2}\left(0, T ; H^{\left.k+1+\delta_{0 k}\right)}\right.}\right) h^{k+1} \\
& +K\left\|\frac{\partial^{2} c}{\partial \tau^{2}}\right\|_{L^{2}\left(0, T ; L^{2}\right)} \Delta t, \\
\max _{0 \leq n \leq N} & \left\|\left(p_{h}-p\right)\left(t^{n}\right)\right\| \\
\leq & K\left(\|c\|_{L^{\infty}\left(0, T ; H^{k+2}\right)}+\left\|c_{t}\right\|_{L^{2}\left(0, T ; H^{\left.k+1+\delta_{0 k}\right)}\right.}\right) h^{k+1} \\
& +K\left\|\frac{\partial^{2} c}{\partial \tau^{2}}\right\|_{L^{2}\left(0, T ; L^{2}\right)} \Delta t .
\end{aligned}
$$

Proof. Lets first show the estimate of (38). Taking $v_{h}=e_{h}^{n}$ and $\chi_{h}=\varepsilon_{h}^{n}$ in (23), we obtain

$$
\begin{aligned}
& \left(\frac{e_{h}^{n}-\bar{e}_{h}^{n-1}}{\Delta t}, e_{h}^{n}\right)+\left(\alpha\left(c_{h}^{n}\right) \varepsilon_{h}^{n}, \varepsilon_{h}^{n}\right) \\
& =\left(\psi \frac{\partial c^{n}}{\partial \tau}-\frac{c^{n}-\bar{c}^{n-1}}{\Delta t}, e_{h}^{n}\right)-\left(\frac{\rho^{n}-\bar{\rho}^{n-1}}{\Delta t}, e_{h}^{n}\right)
\end{aligned}
$$

$$
\begin{aligned}
& +\left(\left(\alpha\left(c^{n}\right)-\alpha\left(c_{h}^{n}\right)\right) \widetilde{p}^{n}, \varepsilon_{h}^{n}\right)+\left(f\left(c_{h}^{n}\right)-f\left(c^{n}\right), e_{h}^{n}\right) \\
= & \sum_{i=1}^{4} I_{i} .
\end{aligned}
$$

For the first term on the right-hand side of the above equation, following the treatment manner in [8], we will bound $\left\|\psi\left(\partial c^{n} / \partial \tau\right)-\left(c^{n}-\bar{c}^{n-1}\right) / \Delta t\right\|$ through the representation below involving an integral in the parameter $\tau$ along the tangent to the characteristics from $\left(\bar{x}, t^{n-1}\right)$ to $\left(x, t^{n}\right)$. Denote the coordinates of the point on the segment by $(x(\tau), t(\tau))$. The standard backward difference quotient error equation is given by

$$
\frac{\partial c^{n}}{\partial t}-\frac{c^{n}-c^{n-1}}{\Delta t}=\frac{1}{\Delta t} \int_{t^{n-1}}^{t^{n}}\left(t-t^{n-1}\right) \frac{\partial^{2} c}{\partial t^{2}} d t
$$

analogously, along the tangent to the characteristics following:

$$
\begin{aligned}
& \psi \frac{\partial c^{n}}{\partial \tau}-\frac{c^{n}-\bar{c}^{n-1}}{\Delta t} \\
& \quad=\frac{1}{\Delta t} \int_{\left(\bar{x}, t^{n-1}\right)}^{\left(x, t^{n}\right)} \sqrt{(x(\tau)-\bar{x})^{2}+\left(t(\tau)-t^{n-1}\right)^{2}} \frac{\partial^{2} c}{\partial \tau^{2}} d \tau .
\end{aligned}
$$

Taking the $L^{2}(\Omega)$ norm of this error term, we obtain

$$
\begin{aligned}
& \left\|\psi \frac{\partial c^{n}}{\partial \tau}-\frac{c^{n}-\bar{c}^{n-1}}{\Delta t}\right\|^{2} \\
& \quad \leq\left\|\psi^{4}\right\|_{0, \infty} \Delta t \int_{\Omega} \int_{t^{n-1}}^{t^{n}}\left|\frac{\partial^{2} c}{\partial \tau^{2}}\left(\frac{t^{n}-t}{\Delta t} \bar{x}+\frac{t-t^{n-1}}{\Delta t} x, t\right)\right|^{2} d t d x,
\end{aligned}
$$

to relate this to a standard norm of $\partial^{2} c / \partial \tau^{2}$, consider the following transformation:

$$
S:(x, t) \longrightarrow(z, t)=\left(\frac{t^{n}-t}{\Delta t} \bar{x}+\frac{t-t^{n-1}}{\Delta t} x, t\right) .
$$

By the assumption of (3), $S$ is invertible for sufficiently small $\Delta t$ and its determinant is $1+O(\Delta t)$. Since our problem is periodic, for any fixed $t \in[0, T], S$ is a homeomorphism of $\Omega$ onto itself, so the same is true for $\Omega \times\left[t^{n-1}, t^{n}\right]$. It follows that

$$
\left\|\psi \frac{\partial c^{n}}{\partial \tau}-\frac{c^{n}-\bar{c}^{n-1}}{\Delta t}\right\|^{2} \leq K\left\|\psi^{4}\right\|_{0, \infty}\left\|\frac{\partial^{2} c}{\partial \tau^{2}}\right\|_{L^{2}\left(t^{n-1}, t^{n} ; L^{2}\right)}^{2} \Delta t,
$$

and the first term on the right-hand side of (40) is bounded by

$$
\left|I_{1}\right| \leq K\left\|\frac{\partial^{2} c}{\partial \tau^{2}}\right\|_{L^{2}\left(t^{n-1}, t^{n} ; L^{2}\right)}^{2} \Delta t+K\left\|e_{h}^{n}\right\|^{2} .
$$

Concerning the second term on the right-hand side of (40), write $\rho^{n}-\bar{\rho}^{n-1}$ as the sum $\left(\rho^{n}-\rho^{n-1}\right)+\left(\rho^{n-1}-\bar{\rho}^{n-1}\right)$, then, 
by Lemma 6 and (23)(b), under the assumption $\Delta t=O(h)$, we have

$$
\begin{aligned}
\left|I_{2}\right| \leq & \frac{K}{\Delta t} \int_{t^{n-1}}^{t^{n}}\left\|\rho_{t}\right\|^{2} d s+K\left\|e_{h}^{n}\right\|^{2} \\
& +K\|\rho\|_{L^{\infty}\left(0, T ; L^{2}\right)}^{2}+\frac{a_{0}}{2}\left\|\varepsilon_{h}^{n}\right\|^{2} .
\end{aligned}
$$

Now we come to the term $I_{3}$, by (4) we have the following:

$$
\left|I_{3}\right| \leq K\left(\left\|e_{h}^{n}\right\|^{2}+\left\|\rho^{n}\right\|^{2}\right)+\frac{a_{0}}{2}\left\|\varepsilon_{h}^{n}\right\|^{2}
$$

About the last term in the right-hand side of (40), by (4), we have

$$
\left|I_{4}\right| \leq K\left(\left\|e_{h}^{n}\right\|^{2}+\left\|\rho^{n}\right\|^{2}\right),
$$

and this completes the treatment of the right-hand side of (40).

By using (33), the left-hand side of (40) is bounded by $(1 / 2 \Delta t)\left[\left(e_{h}^{n}, e_{h}^{n}\right)-(1+K \Delta t)\left(e_{h}^{n-1}, e_{h}^{n-1}\right)\right]+a_{0}\left\|\varepsilon_{h}^{n}\right\|^{2}$, combining (46)-(49) with (40) to give the following recursion relation:

$$
\begin{aligned}
\frac{1}{2 \Delta t}\left[\left(e_{h}^{n}, e_{h}^{n}\right)-\left(e_{h}^{n-1}, e_{h}^{n-1}\right)\right]+a_{0}\left\|\varepsilon_{h}^{n}\right\|^{2} \\
\leq K \Delta t\left\|\frac{\partial^{2} c}{\partial \tau^{2}}\right\|_{L^{2}\left(t^{n-1}, t^{n} ; L^{2}\right)}^{2} \\
+\frac{K}{\Delta t} \int_{t^{n-1}}^{t^{n}}\left\|\rho_{t}\right\|^{2} d s+K\|\rho\|_{L^{\infty}\left(0, T ; L^{2}\right)}^{2} \\
+K\left\|e_{h}^{n}\right\|^{2}+K\left\|e_{h}^{n-1}\right\|^{2}+a_{0}\left\|\varepsilon_{h}^{n}\right\|^{2} .
\end{aligned}
$$

If (50) is multiplied by $2 \Delta t$ and summed in time, and if it is noted that $(10)(\mathrm{c})$ implies that $e_{h}^{0}=0$, then, it follows that

$$
\begin{aligned}
\left\|e_{h}^{n}\right\|^{2} \leq & K\left(\Delta t^{2}\left\|\frac{\partial^{2} c}{\partial \tau^{2}}\right\|_{L^{2}\left(0, T ; L^{2}\right)}^{2}\right. \\
& \left.+\left\|\rho_{t}\right\|_{L^{2}\left(0, T ; L^{2}\right)}^{2}+\|\rho\|_{L^{\infty}\left(0, T ; L^{2}\right)}^{2}\right) \\
& +K \Delta t \sum_{i=1}^{n}\left\|e_{h}^{i}\right\|^{2} .
\end{aligned}
$$

For sufficiently small $\Delta t>0$, by Gronwall's lemma, we obtain

$$
\left\|e_{h}^{n}\right\| \leq K\left(\left\|\rho_{t}\right\|_{L^{2}\left(0, T ; L^{2}\right)}+\|\rho\|_{L^{\infty}\left(0, T ; L^{2}\right)}\right)+K \Delta t\left\|\frac{\partial^{2} c}{\partial \tau^{2}}\right\|_{L^{2}\left(0, T ; L^{2}\right)} .
$$

Recall that the error $c_{h}-c=e_{h}+\rho,(19),(21)$, and (53) together imply that

$$
\begin{aligned}
\max _{0 \leq n \leq N} & \left\|\left(c_{h}-c\right)\left(t^{n}\right)\right\| \\
\leq & K\left(\|c\|_{L^{\infty}\left(0, T ; H^{\left.k+1+\delta_{0 k}\right)}\right.}+\left\|c_{t}\right\|_{L^{2}\left(0, T ; H^{\left.k+1+\delta_{0 k}\right)}\right.}\right) h^{k+1} \\
& +K\left\|\frac{\partial^{2} c}{\partial \tau^{2}}\right\|_{L^{2}\left(0, T ; L^{2}\right)} \Delta t .
\end{aligned}
$$

This completes the first part of our conclusion.
In order to show the estimate (39), by (23)(b), we get

$$
\begin{aligned}
& \left(\frac{\alpha\left(c_{h}^{n}\right) \varepsilon_{h}^{n}-\alpha\left(c_{h}^{n-1}\right) \varepsilon_{h}^{n-1}}{\Delta t}, \chi_{h}\right)+\left(\frac{e_{h}^{n}-e_{h}^{n-1}}{\Delta t}, \operatorname{div} \chi_{h}\right) \\
& \quad=\left(\frac{\left(\alpha\left(c^{n}\right)-\alpha\left(c_{h}^{n}\right)\right) \widetilde{p}^{n}-\left(\alpha\left(c^{n-1}\right)-\alpha\left(c_{h}^{n-1}\right)\right) \widetilde{p}^{n-1}}{\Delta t}, \chi_{h}\right) .
\end{aligned}
$$

Choosing $v_{h}=\left(e_{h}^{n}-e_{h}^{n-1}\right) / \Delta t$ and $\chi_{h}=\varepsilon_{h}^{n}$, respectively, in (23)(a) and (54) and adding them to obtain

$$
\begin{aligned}
&\left(\frac{e_{h}^{n}-e_{h}^{n-1}}{\Delta t}, \frac{e_{h}^{n}-e_{h}^{n-1}}{\Delta t}\right)+\left(\frac{\alpha\left(c_{h}^{n}\right) \varepsilon_{h}^{n}-\alpha\left(c_{h}^{n-1}\right) \varepsilon_{h}^{n-1}}{\Delta t}, \varepsilon_{h}^{n}\right) \\
&=\left(\psi \frac{\partial c^{n}}{\partial \tau}-\frac{c^{n}-\bar{c}^{n-1}}{\Delta t}, \frac{e_{h}^{n}-e_{h}^{n-1}}{\Delta t}\right) \\
&-\left(\frac{\rho^{n}-\rho^{n-1}}{\Delta t}, \frac{e_{h}^{n}-e_{h}^{n-1}}{\Delta t}\right) \\
&-\left(\frac{\rho^{n-1}-\bar{\rho}^{n-1}}{\Delta t}, \frac{e_{h}^{n}-e_{h}^{n-1}}{\Delta t}\right) \\
&+\left(\frac{\left(\alpha\left(c^{n}\right)-\alpha\left(c_{h}^{n}\right)\right) \widetilde{p}^{n}-\left(\alpha\left(c^{n-1}\right)-\alpha\left(c_{h}^{n-1}\right)\right) \widetilde{p}^{n-1}}{\Delta t}, \varepsilon_{h}^{n}\right) \\
&-\left(\frac{e_{h}^{n-1}-\bar{e}_{h}^{n-1}}{\Delta t}, \frac{e_{h}^{n}-e_{h}^{n-1}}{\Delta t}\right) \\
&+\left(f\left(c_{h}^{n}\right)-f\left(c^{n}\right), \frac{e_{h}^{n}-e_{h}^{n-1}}{\Delta t}\right) .
\end{aligned}
$$

The left-hand side satisfies the inequality:

$$
\begin{gathered}
\left(\frac{e_{h}^{n}-e_{h}^{n-1}}{\Delta t}, \frac{e_{h}^{n}-e_{h}^{n-1}}{\Delta t}\right)+\left(\frac{\alpha\left(c_{h}^{n}\right) \varepsilon_{h}^{n}-\alpha\left(c_{h}^{n-1}\right) \varepsilon_{h}^{n-1}}{\Delta t}, \varepsilon_{h}^{n}\right) \\
\geq \frac{1}{2 \Delta t}\left(\left(\alpha\left(c_{h}^{n}\right) \varepsilon_{h}^{n}, \varepsilon_{h}^{n}\right)-\left(\alpha\left(c_{h}^{n-1}\right) \varepsilon_{h}^{n-1}, \varepsilon_{h}^{n-1}\right)\right) \\
\quad+\frac{1}{2 \Delta t}\left(\left(\alpha\left(c_{h}^{n-1}\right)-\alpha\left(c_{h}^{n}\right)\right) \varepsilon_{h}^{n-1}, \varepsilon_{h}^{n-1}\right) \\
+\frac{1}{\Delta t}\left(\left(\alpha\left(c_{h}^{n}\right)-\alpha\left(c_{h}^{n-1}\right)\right) \varepsilon_{h}^{n-1}, \varepsilon_{h}^{n}\right)\left\|\frac{e_{h}^{n}-e_{h}^{n-1}}{\Delta t}\right\|^{2}
\end{gathered}
$$

Substitute it into (55), we have

$$
\begin{aligned}
& \left\|\frac{e_{h}^{n}-e_{h}^{n-1}}{\Delta t}\right\|^{2}+\frac{1}{2 \Delta t}\left(\left(\alpha\left(c_{h}^{n}\right) \varepsilon_{h}^{n}, \varepsilon_{h}^{n}\right)-\left(\alpha\left(c_{h}^{n-1}\right) \varepsilon_{h}^{n-1}, \varepsilon_{h}^{n-1}\right)\right) \\
& =\left(\psi \frac{\partial c^{n}}{\partial \tau}-\frac{c^{n}-\bar{c}^{n-1}}{\Delta t}, \frac{e_{h}^{n}-e_{h}^{n-1}}{\Delta t}\right) \\
& +\left(\frac{\left(\alpha\left(c^{n}\right)-\alpha\left(c_{h}^{n}\right)\right) \widetilde{p}^{n}-\left(\alpha\left(c^{n-1}\right)-\alpha\left(c_{h}^{n-1}\right)\right) \widetilde{p}^{n-1}}{\Delta t}, \varepsilon_{h}^{n}\right) \\
& +\frac{1}{2 \Delta t}\left(\left(\alpha\left(c_{h}^{n-1}\right)-\alpha\left(c_{h}^{n}\right)\right) \varepsilon_{h}^{n-1}, 2 \varepsilon_{h}^{n}-\varepsilon_{h}^{n-1}\right)
\end{aligned}
$$


Discrete Dynamics in Nature and Society

7

$$
\begin{aligned}
& +\left(f\left(c_{h}^{n}\right)-f\left(c^{n}\right), \frac{e_{h}^{n}-e_{h}^{n-1}}{\Delta t}\right) \\
& -\left(\frac{\rho^{n}-\rho^{n-1}}{\Delta t}, \frac{e_{h}^{n}-e_{h}^{n-1}}{\Delta t}\right)-\left(\frac{\rho^{n-1}-\bar{\rho}^{n-1}}{\Delta t}, \frac{e_{h}^{n}-e_{h}^{n-1}}{\Delta t}\right) \\
& -\left(\frac{e_{h}^{n-1}-\bar{e}_{h}^{n-1}}{\Delta t}, \frac{e_{h}^{n}-e_{h}^{n-1}}{\Delta t}\right) \\
& =\sum_{i=1}^{7} J_{i} .
\end{aligned}
$$

Bound $J_{1}-J_{5}$ on the right-hand side of (56) one by one, we have

$$
\begin{aligned}
\left|J_{1}\right| \leq & K\left\|\frac{\partial^{2} c}{\partial \tau^{2}}\right\|_{L^{2}\left(t^{n-1}, t^{n} L^{2}\right)} \Delta t+\frac{1}{6}\left\|\frac{e_{h}^{n}-e_{h}^{n-1}}{\Delta t}\right\|^{2}, \\
\left|J_{2}\right| \leq & K\left(\left\|e_{h}\right\|^{2}+\left\|\rho^{n}\right\|^{2}+\left\|e_{h}^{n-1}\right\|^{2}+\left\|\rho^{n-1}\right\|^{2}\right) \\
& +\frac{1}{6}\left\|\frac{e_{h}^{n}-e_{h}^{n-1}}{\Delta t}\right\|^{2} \\
& +\frac{K}{\Delta t} \int_{t^{n-1}}^{t^{n}}\left\|\rho_{t}\right\|^{2} d t+K\left\|\varepsilon_{h}^{n}\right\|^{2}, \\
\left|J_{3}\right| \leq & K\left(\left\|\varepsilon_{h}^{n-1}\right\|_{0, \infty}^{2}+1\right)\left(\left\|\varepsilon_{h}^{n}\right\|^{2}+\left\|\varepsilon_{h}^{n-1}\right\|^{2}\right) \\
& +\frac{1}{6}\left\|\frac{e_{h}^{n}-e_{h}^{n-1}}{\Delta t}\right\|^{2} .
\end{aligned}
$$

Concerning $J_{4}$ and $J_{5}$, we can easily get

$$
\begin{gathered}
\left|J_{4}\right| \leq K\left(\left\|e_{h}^{n}\right\|^{2}+\left\|\rho^{n}\right\|^{2}\right)+\frac{1}{6}\left\|\frac{e_{h}^{n}-e_{h}^{n-1}}{\Delta t}\right\|^{2}, \\
\left|J_{5}\right| \leq \frac{K}{\Delta t} \int_{t^{n-1}}^{t^{n}}\left\|\rho_{t}\right\|^{2} d t+\frac{1}{6}\left\|\frac{e_{h}^{n}-e_{h}^{n-1}}{\Delta t}\right\|^{2} .
\end{gathered}
$$

Substituting (58)-(59) into (57) to obtain

$$
\begin{aligned}
\left\|\frac{e_{h}^{n}-e_{h}^{n-1}}{\Delta t}\right\|^{2}+\frac{1}{2 \Delta t}\left(\left(\alpha\left(c_{h}^{n}\right) \varepsilon_{h}^{n}, \varepsilon_{h}^{n}\right)-\left(\alpha\left(c_{h}^{n-1}\right) \varepsilon_{h}^{n-1}, \varepsilon_{h}^{n-1}\right)\right) \\
\leq K\left\|\frac{\partial^{2} c}{\partial \tau^{2}}\right\|_{L^{2}\left(t^{n-1}, t^{n} ; L^{2}\right)}^{2} \Delta t \\
+K\left(\left\|e_{h}^{n}\right\|^{2}+\left\|\rho^{n}\right\|^{2}+\left\|e_{h}^{n-1}\right\|^{2}+\left\|\rho^{n-1}\right\|^{2}\right) \\
+\frac{5}{6}\left\|\frac{e_{h}^{n}-e_{h}^{n-1}}{\Delta t}\right\|^{2}+\frac{K}{\Delta t} \int_{t^{n-1}}^{t^{n}}\left\|\rho_{t}\right\|^{2} d t \\
+K\left(1+\left\|\varepsilon_{h}^{n-1}\right\|_{0, \infty}^{2}\right)\left(\left\|\varepsilon_{h}^{n}\right\|^{2}+\left\|\varepsilon_{h}^{n-1}\right\|^{2}\right)
\end{aligned}
$$

$$
\begin{aligned}
& -\left(\frac{\rho^{n-1}-\bar{\rho}^{n-1}}{\Delta t}, \frac{e_{h}^{n}-e_{h}^{n-1}}{\Delta t}\right) \\
& -\left(\frac{e_{h}^{n-1}-\bar{e}_{h}^{n-1}}{\Delta t}, \frac{e_{h}^{n}-e_{h}^{n-1}}{\Delta t}\right) .
\end{aligned}
$$

Noting that $e_{h}^{0}=0$, we have $\left\|\varepsilon_{h}^{0}\right\| \leq K\left\|\rho^{0}\right\|$ from (23)(b). To multiply (60) by $2 \Delta t$ and add it in time, we obtain

$$
\begin{gathered}
\frac{\Delta t}{3} \sum_{i=1}^{n}\left\|\frac{e_{h}^{n}-e_{h}^{n-1}}{\Delta t}\right\|^{2}+a_{0}\left\|\varepsilon_{h}^{n}\right\|^{2} \\
\leq K\left\|\frac{\partial^{2} c}{\partial \tau^{2}}\right\|_{L^{2}\left(0, T ; L^{2}\right)}^{2} \Delta t^{2} \\
+K \Delta t\left(1+\max _{0 \leq i \leq n-1}\left\|\varepsilon_{h}^{i}\right\|_{0, \infty}\right) \sum_{i=1}^{n}\left\|\varepsilon_{h}^{i}\right\|^{2} \\
+K\left(\|\rho\|_{L^{\infty}}^{2}\left(0, T ; L^{2}\right)+\left\|\rho_{t}\right\|_{L^{2}\left(0, T ; L^{2}\right)}^{2}\right. \\
\left.+\max _{1 \leq i \leq n}\left\|e_{h}^{i}\right\|^{2}+\left\|\rho^{0}\right\|^{2}\right) \\
-2\left(\sum_{i=1}^{n}\left(\frac{\rho^{i-1}-\bar{\rho}^{i-1}}{\Delta t}, e_{h}^{i}-e_{h}^{i-1}\right)\right. \\
\left.+\sum_{i=1}^{n}\left(\frac{e_{h}^{i-1}-\bar{e}_{h}^{i-1}}{\Delta t}, e_{h}^{i}-e_{h}^{i-1}\right)\right) .
\end{gathered}
$$

By (23)(b) and Lemma 6, we have

$$
\begin{aligned}
\sum_{i=1}^{n}( & \left.\frac{\rho^{i-1}-\bar{\rho}^{i-1}}{\Delta t}, e_{h}^{i}-e_{h}^{i-1}\right) \\
\leq & K\left(\max _{1 \leq i \leq n}\left\|e_{h}^{i}\right\|^{2}+\|\rho\|_{L^{\infty}\left(0, T ; L^{2}\right)}^{2}+\left\|\rho_{t}\right\|_{L^{2}\left(0, T ; L^{2}\right)}^{2}\right) \\
& +\frac{a_{0}}{4}\left\|\varepsilon_{h}^{n}\right\|^{2}+K \Delta t \sum_{i=1}^{n-1}\left\|\varepsilon_{h}^{i}\right\|^{2},
\end{aligned}
$$

where the assumption $\Delta t=O(h)$ is used. Similarly, we get

$$
\begin{aligned}
\sum_{i=1}^{n}\left(\frac{e_{h}^{i-1}-\bar{e}_{h}^{i-1}}{\Delta t}, e_{h}^{i}-e_{h}^{i-1}\right) \\
\leq K\left(\max _{1 \leq i \leq n}\left\|e_{h}^{i}\right\|^{2}+\|\rho\|_{L^{\infty}\left(0, T ; L^{2}\right)}^{2}\right)+\frac{a_{0}}{4}\left\|\varepsilon_{h}^{n}\right\|^{2} \\
+K \Delta t \sum_{i=1}^{n-1}\left\|\varepsilon_{h}^{i}\right\|^{2}+\frac{\Delta t}{3} \sum_{i=1}^{n-1}\left\|\frac{e_{h}^{i}-e_{h}^{i-1}}{\Delta t}\right\|^{2}
\end{aligned}
$$


Substituting (62) and (63) into (61), we get

$$
\begin{aligned}
\Delta t \sum_{i=1}^{n}\left\|\frac{e_{h}^{i}-e_{h}^{i-1}}{\Delta t}\right\|^{2}+a_{0}\left\|\varepsilon_{h}^{n}\right\|^{2} \\
\leq K \Delta t\left(1+\max _{0 \leq i \leq n}\left\|\varepsilon_{h}^{i}\right\|_{0, \infty}\right) \sum_{i=1}^{n}\left\|\varepsilon_{h}^{i}\right\|^{2} \\
\quad+K \Delta t^{2}\left\|\frac{\partial^{2} c}{\partial \tau^{2}}\right\|_{L^{2}\left(0, T ; L^{2}\right)}^{2} \\
\quad+K\left(\left\|\rho_{t}\right\|_{L^{2}\left(0, T ; L^{2}\right)}^{2}+\|\rho\|_{L^{\infty}\left(0, T ; L^{2}\right)}^{2}+\max _{1 \leq i \leq n}\left\|e_{h}^{i}\right\|^{2}\right) .
\end{aligned}
$$

We see that an application of Gronwall's lemma would complete the second part of our argument if we did not have the term $\max _{0 \leq i \leq n-1}\left\|\varepsilon_{h}^{i}\right\|_{0, \infty}$ on the right hand side of (64). We will use an induction argument to prove the hypothesis as follows:

$$
\left\|\varepsilon_{h}^{i}\right\|_{0, \infty} \leq M_{1}, \quad i=1,2, \ldots, N
$$

Since $\left\|\varepsilon_{h}^{0}\right\| \leq K\left\|\rho^{0}\right\|$, by the inverse property and (19), it can be easily verified that $\left\|\varepsilon_{h}^{0}\right\|_{0, \infty} \leq M_{1}$. Using (19), (21), (52), and (64) we see that (65) clearly holds for $i=1$ if $\Delta t$ is sufficiently small. Assume that (65) holds for $i=0,1, \ldots, n-1$, using (64), we obtain

$$
\begin{aligned}
\left\|\varepsilon_{h}^{n}\right\|^{2} \leq & K \Delta t \sum_{i=1}^{n}\left\|\varepsilon_{h}^{i}\right\|^{2} \\
& +K\left\|\frac{\partial^{2} c}{\partial \tau^{2}}\right\|_{L^{2}\left(0, T ; L^{2}\right)}^{2} \Delta t^{2} \\
& +K\left(\max _{1 \leq i \leq n}\left\|e_{h}^{i}\right\|^{2}+\left\|\rho_{t}\right\|_{L^{2}\left(0, T ; L^{2}\right)}^{2}+\|\rho\|_{L^{\infty}\left(0, T ; L^{2}\right)}^{2}\right),
\end{aligned}
$$

Then, an application of Gronwall's lemma gives us an estimate which together with (19), (21), and (52) and the inverse property of $H_{h}$, show that the induction hypothesis (65) holds for $i=n$ for sufficiently small $\Delta t$. This completes the induction argument.

Finally by Gronwall's lemma, it follows from (64) that

$$
\begin{aligned}
\left\|\varepsilon_{h}^{n}\right\| \leq & K\left(\left\|\rho_{t}\right\|_{L^{2}\left(0, T ; L^{2}\right)}+\|\rho\|_{L^{\infty}\left(0, T ; L^{2}\right)}+\max _{1 \leq i \leq n}\left\|e_{h}^{i}\right\|\right) \\
& +K\left\|\frac{\partial^{2} c}{\partial \tau^{2}}\right\|_{L^{2}\left(0, T ; L^{2}\right)} \Delta t .
\end{aligned}
$$

Note that $p_{h}-p=\varepsilon_{h}+\sigma$, by (52) and (19)-(21), we obtain

$$
\begin{aligned}
\| p_{h}^{n}- & p^{n} \| \\
\leq & K\left(\left\|c_{t}\right\|_{L^{2}\left(0, T ; H^{\left.k+1+\delta_{0 k}\right)}\right.}+\|c\|_{L^{\infty}\left(0, T ; H^{k+2}\right)}\right) h^{k+1} \\
& +K\left\|\frac{\partial^{2} c}{\partial \tau^{2}}\right\|_{L^{2}\left(0, T ; L^{2}\right)} \Delta t .
\end{aligned}
$$

This also shows that

$$
\begin{aligned}
\max _{0 \leq n \leq N} & \left\|p_{h}^{n}-p^{n}\right\| \\
\leq & K\left(\left\|c_{t}\right\|_{L^{2}\left(0, T ; H^{\left.k+1+\delta_{0 k}\right)}\right.}+\|c\|_{L^{\infty}\left(0, T ; H^{k+2}\right)}\right) h^{k+1} \\
& +K\left\|\frac{\partial^{2} c}{\partial \tau^{2}}\right\|_{L^{2}\left(0, T ; L^{2}\right)} \Delta t .
\end{aligned}
$$

Note that our results in this paper do not cover the cases of nonlinear convection-dominated systems, which are of importance in some applications, particularly in reservoir simulations.

\section{Acknowledgment}

This research is partially supported by the China Postdoctoral Science Foundation funded project (2011M501149), the Humanity and Social Science Foundation of the Ministry of Education of China (12YJCZH303), the Special Fund Project for Post Doctoral Innovation of Shandong Province (201103061), and Independent Innovation Foundation of Shandong University, IIFSDU (IFW12109).

\section{References}

[1] M. R. Todd, P. M. O’Dell, and G. J. Hirasaki, "Methods for increased accuracy in numerical reservoir simulators," Society of Petroleum Engineers Journal, vol. 12, no. 6, pp. 515-530, 1972.

[2] G. Chavent and J. Jaffre, Mathematical Models and Finite Elements For Reservoir Simulation, Elsevier, New York, NY, USA, 1986.

[3] F. Miniati and P. Colella, "A modified higher order Godunov's scheme for stiff source conservative hydrodynamics," Journal of Computational Physics, vol. 224, no. 2, pp. 519-538, 2007.

[4] Z. Si, X. Feng, and A. Abduwali, "The semi-discrete streamline diffusion finite element method for time-dependented convection-diffusion problems," Applied Mathematics and Computation, vol. 202, no. 2, pp. 771-779, 2008.

[5] D. Yang, "Some least-squares Galerkin procedures for firstorder time-dependent convection-diffusion system," Computer Methods in Applied Mechanics and Engineering, vol. 180, no. 1-2, pp. 81-95, 1999.

[6] D.-P. Yang, "Analysis of least-squares mixed finite element methods for nonlinear nonstationary convection-diffusion problems," Mathematics of Computation, vol. 69, no. 231, pp. 929-963, 2000.

[7] J. Douglas, Jr. and T. F. Russell, "Numerical methods for convection-dominated diffusion problems based on combining the method of characteristics with finite element or finite difference procedures," SIAM Journal on Numerical Analysis, vol. 19, no. 5, pp. 871-885, 1982.

[8] R. E. Ewing, T. F. Russell, and M. F. Wheeler, "Convergence analysis of an approximation of miscible displacement in porous media by mixed finite elements and a modified method of characteristics," Computer Methods in Applied Mechanics and Engineering, vol. 47, no. 1-2, pp. 73-92, 1984. 
[9] C. N. Dawson, T. F. Russell, and M. F. Wheeler, "Some improved error estimates for the modified method of characteristics," SIAM Journal on Numerical Analysis, vol. 26, no. 6, pp. 1487$1512,1989$.

[10] M. A. Celia, T. F. Russell, I. Herrera, and R. E. Ewing, "An Eulerian-Lagrangian localized adjoint method for the advection-diffusion equation," Advances in Water Resources, vol. 13, no. 4, pp. 187-206, 1990.

[11] J. Douglas, Jr. and J. E. Roberts, "Global estimates for mixed methods for second order elliptic equations," Mathematics of Computation, vol. 44, no. 169, pp. 39-52, 1985.

[12] Y. Chen and Z. Lu, "Error estimates for parabolic optimal control problem by fully discrete mixed finite element methods," Finite Elements in Analysis and Design, vol. 46, no. 11, pp. 957965, 2010.

[13] F. A. Milner and E.-J. Park, "A mixed finite element method for a strongly nonlinear second-order elliptic problem," Mathematics of Computation, vol. 64, no. 211, pp. 973-988, 1995.

[14] K.-Y. Kim, "Guaranteed a posteriori error estimator for mixed finite element methods of elliptic problems," Applied Mathematics and Computation, vol. 218, no. 24, pp. 11820-11831, 2012.

[15] P.-A. Raviart and J. M. Thomas, "A mixed finite element method for 2nd order elliptic problems," in Mathematical Aspects of Finite Element Methods, vol. 606 of Lecture Notes in Mathematics, pp. 292-315, Springer, Berlin, Germany, 1977. 


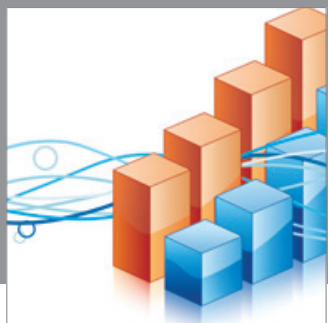

Advances in

Operations Research

mansans

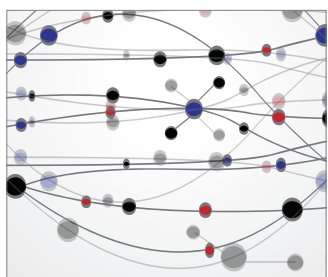

The Scientific World Journal
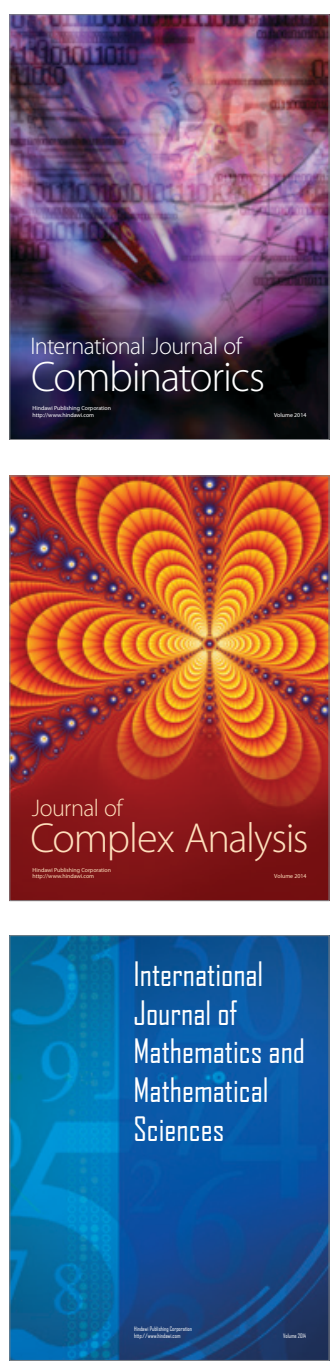
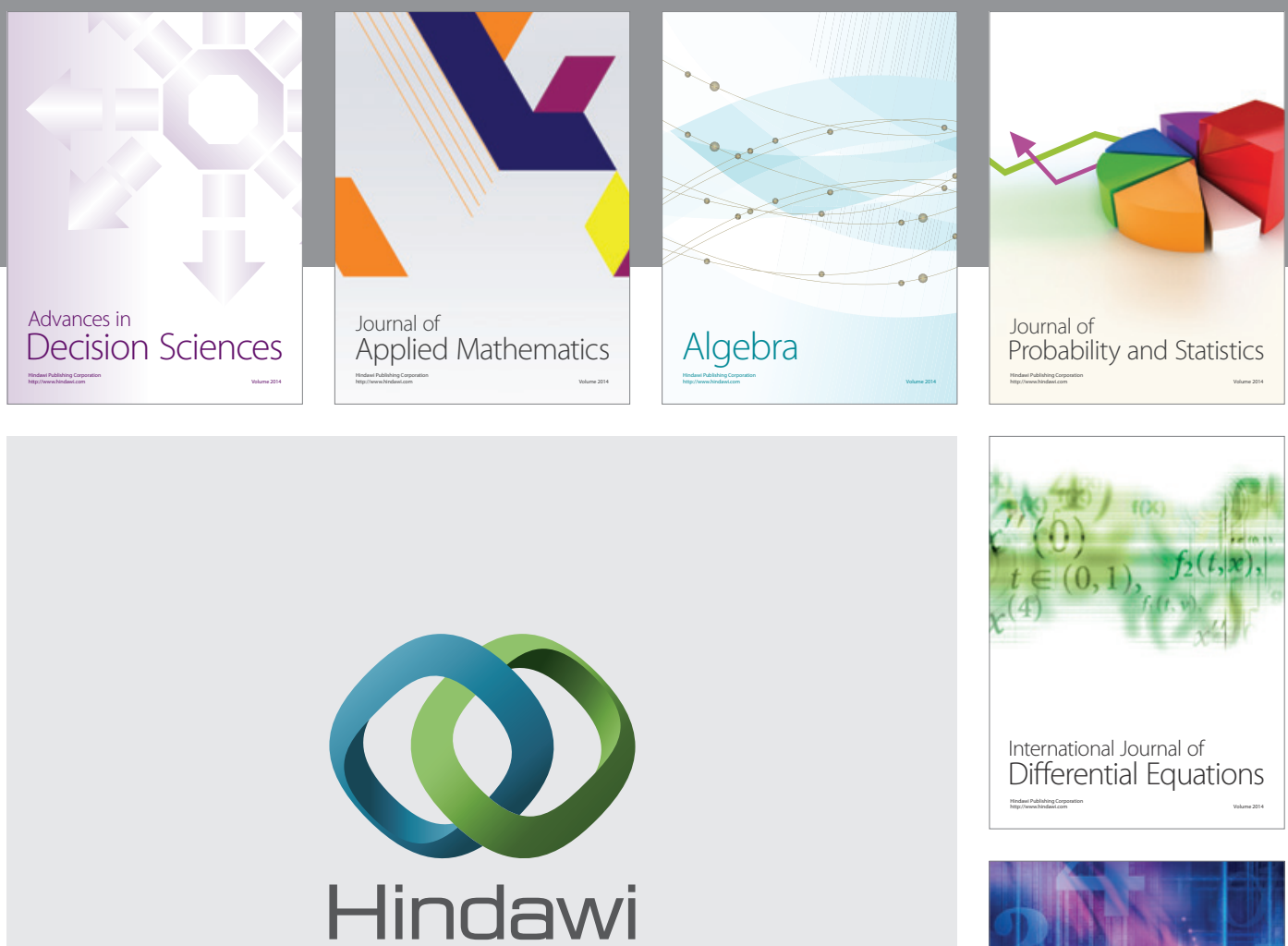

Submit your manuscripts at http://www.hindawi.com
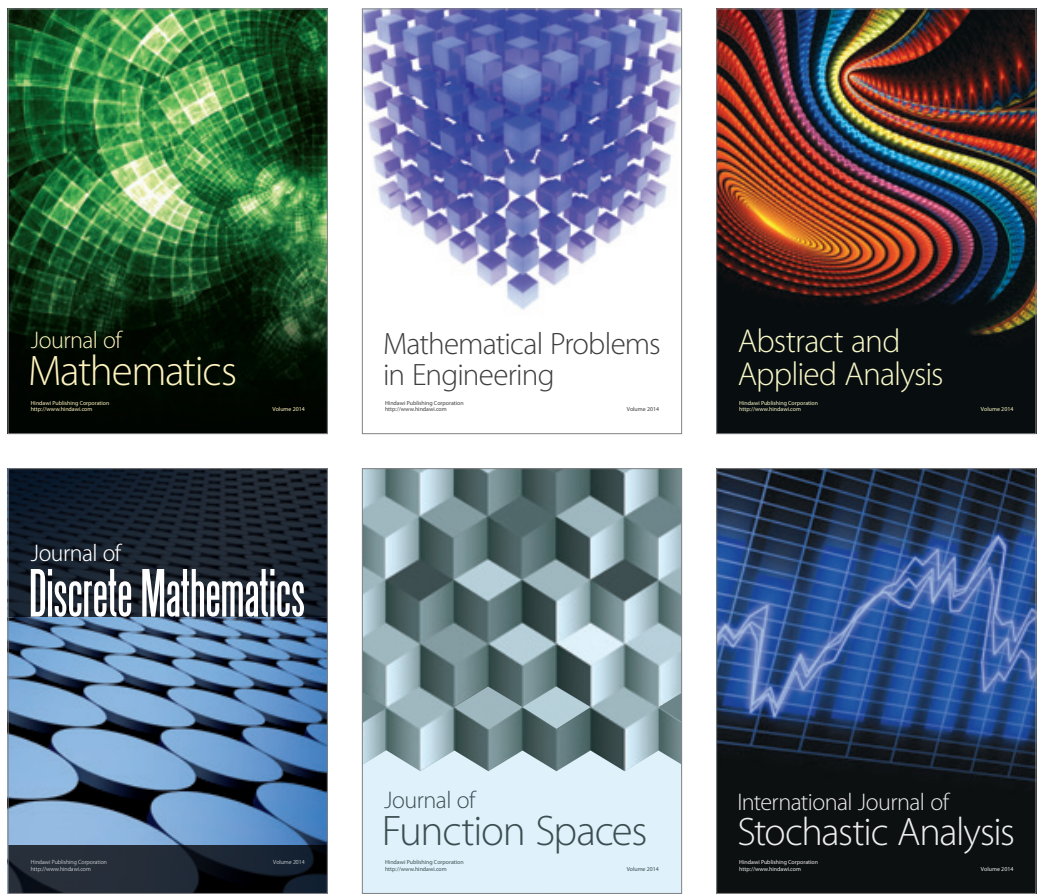

Journal of

Function Spaces

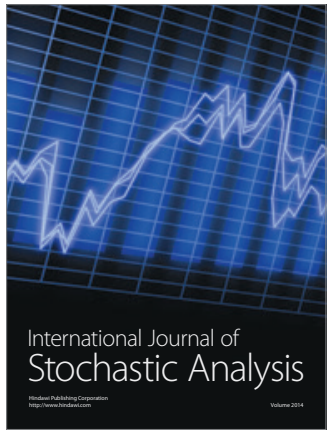

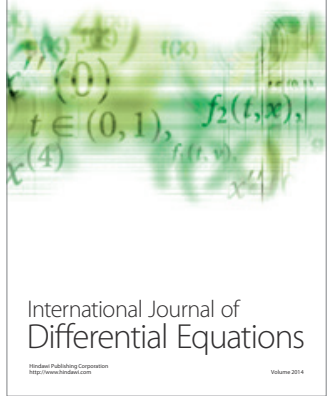
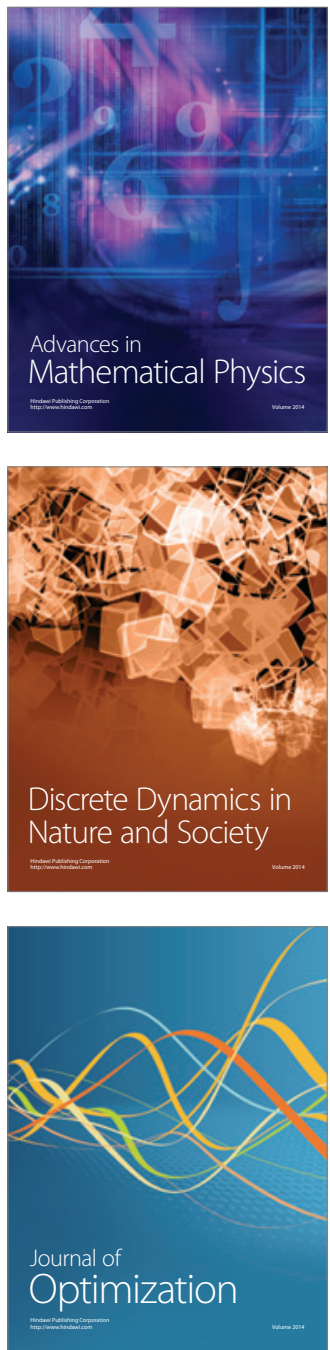